\title{
Anomalous origin of the left coronary artery from the pulmonary artery with abundant collateral vessels
}

\author{
Xiaojing $\mathrm{Ma}^{1,2,3}$ and Guoying Huang ${ }^{1,2,3 *}$ \\ ${ }^{1}$ Heart Center, Children's Hospital of Fudan University, Shanghai, China \\ ${ }^{2}$ National Children's Medical Center, Shanghai, China \\ ${ }^{3}$ Shanghai Key Laboratory of Birth Defects, Shanghai, China
}

\begin{abstract}
Anomalous left coronary artery from the pulmonary artery (ALCAPA) is a rare but potentially life-threatening congenital heart defect. ALCAPA with abundant collateral vessels tends to be misdiagnosed. We present a case of ALCAPA with abundant collateral vessels manifested as a normal heart size and cardiac function, focusing on the importance of awareness of this situation.
\end{abstract}

\begin{abstract}
Abbreviations: ALCAPA: Anomalous Origin of the Left Coronary Artery from the Pulmonary Artery; CHD: Congenital Heart Disease

\section{Introduction}

Anomalous left coronary artery from the pulmonary artery (ALCAPA) is a rare but potentially life-threatening congenital heart defect. The incidence of ALCAPA is 1 in 300,000 live births. The mortality in untreated individuals is high during the first year of life, and only $10-15 \%$ of the patients reach adulthood [1]. In the infants with ALCAPA, the left coronary artery (LCA) receives deoxygenated blood via the pulmonary artery. In elder patients, the right coronary artery (RCA) supplies blood to the LCA territory through a network of collaterals, which causes a coronary steal phenomenon, because preferential blood flow into the low-pressure pulmonary system rather than into the high-resistance myocardium. The above situations will both lead to myocardial ischemia. So, the combination of cardiomegaly and heart failure is a very common finding in ALCAPA, which may lead to the erroneous diagnosis of myocarditis or dilated cardiomyopathy $[2,3]$. Silverman [4] suggested that every infant under 2 years of age referred with a diagnosis of dilated cardiomyopathy should have the diagnosis of ALCAPA excluded. However, a normal heart size and cardiac function does not rule out the diagnosis of ALCAPA. Here, we present a case of ALCAPA with abundant collateral vessels.
\end{abstract}

\section{Case}

A 7-year-old girl with chest pain was admitted. Her creatine kinase isoenzyme, troponin I level and other laboratory tests were within the normal range. Chest X-ray and electrocardiogram revealed no obvious abnormality. Echocardiography suggested the LCA and RCA dilated, and the dimensions of the cardiac chambers and the left ventricular ejection fraction were within the normal ranges (Figure 1). Singlephoton emission computed tomography (SPECT) demonstrated myocardial ischemia in the left ventricular inferior wall and the base of anterior wall (Figure 2). Coronary artery CTA revealed the LCA and RCA dilated. For further evaluation, a coronary angiography was performed. The diagnosis of ALCAPA was made based on the signs that the contrast agent from the RCA through the collateral vessels into the LCA, and finally into the pulmonary artery by the selective right coronary angiography (Figure 3). The patient was subjected to the surgery, direct reimplantation of the LCA onto the aorta. A series of follow-up until 6 months after surgery suggested satisfactory results without any more chest pain. Color Doppler imaging on echocardiography could display the blood flow from aorta into the LCA.

There are two types of ALCAPA. It is termed the adult type if the collateral vessels between the normal RCA and abnormal LCA are well established, the infant type if there are no or few collateral vessels. We are relatively familiar with the infant type, which manifested as cardiomegaly, left ventricular dysfunction and mitral regurgitation, like dilated cardiomyopathy. The adult type is even more unusual, accounting for $10-15 \%$ of all cases of ALCAPA [1]. Patients with an adequate collateral supply may be associated with normal heart size and better cardiac function and can remain asymptomatic until adolescence or adulthood [5-7]. So, the awareness of the varied clinical manifestations of ALCAPA is important. Echocardiography is the firstline imaging method in the diagnosis of ALCAPA. However, there are inherent limitations of echocardiography that reduce the ability to distinguish the origin of the coronary artery with confidence, therefore, the diagnosis of ALCAPA can be ruled in, but never be ruled out, by echocardiography [8]. In our case, the ultrasonic dropout artifact of the wall of the aorta adjacent to the transverse sinus and the wall of the LCA misidentified as the origin of LCA. Color flow mapping is helpful, but also has limitations. It is easy to cause our vigilance when the blood reversely flows from the pulmonary artery to the LCA. But we are apt to

*Correspondence to: Guoying Huang, MD, Heart Center, Children's Hospital of Fudan University, 399 Wanyuan Road, Shanghai, Shanghai 201102, China, Tel: +86-21-64931928; E-mail: gyhuang2010@aliyun.com

Key words: echocardiography, coronary angiography

Received: May 08, 2019; Accepted: May 20, 2019; Published: May 23, 2019 

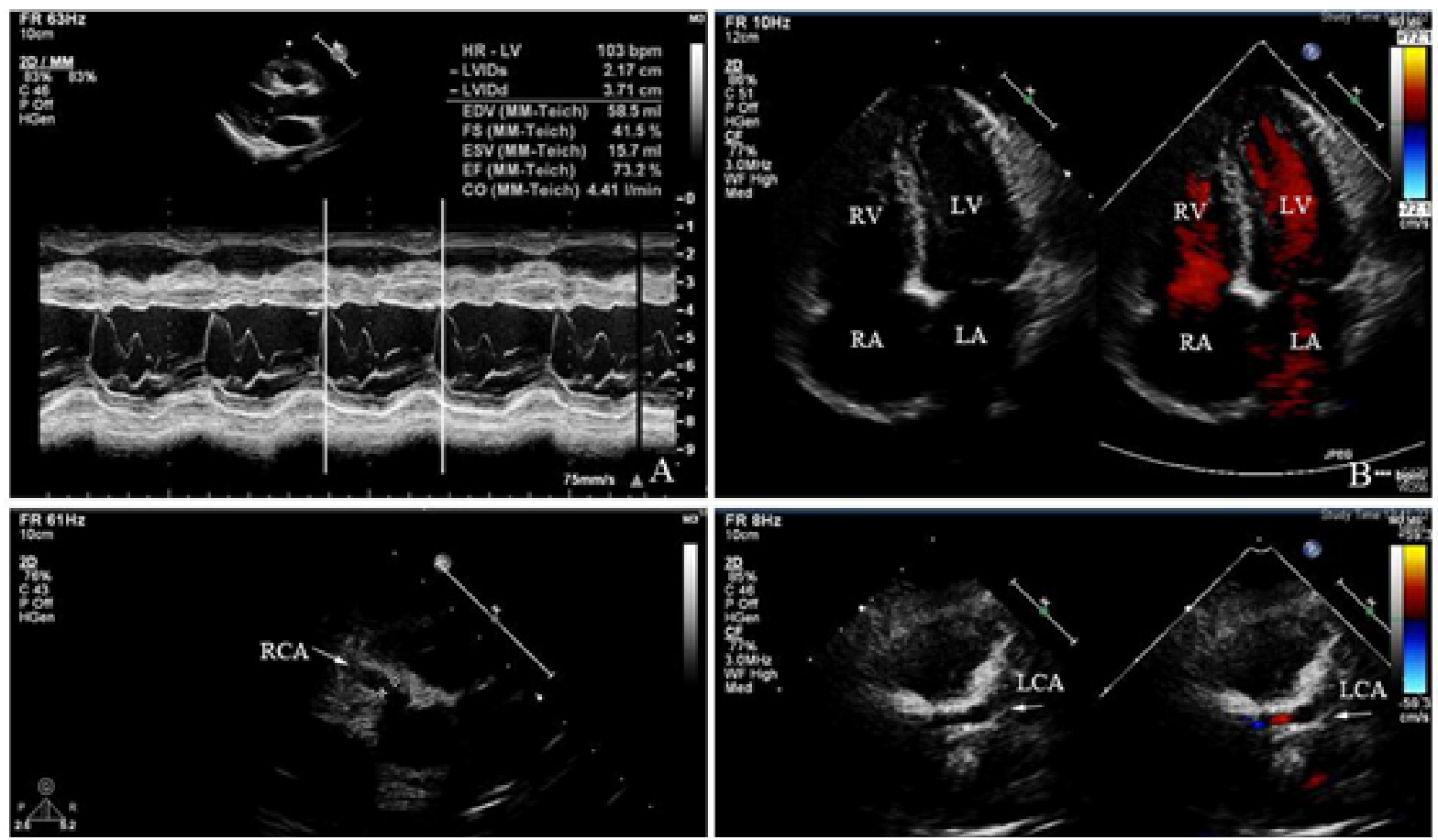

Figure 1. A: M-mode echocardiography revealed the normal size of left ventricle, and the ejection fraction was within the normal range. B: Apical four chamber view revealed the normal size of the atria and the ventricles by two-dimensional echocardiography. C: Echocardiography suggested the dilated right coronary artery. D: Echocardiography suggested the dilated left coronary artery, and color Doppler imaging showing the flow in the left coronary artery.

LV: left ventricle; RV: right ventricle; LA: left atrium; RA: right atrium; RCA: right coronary artery; LCA: left coronary artery.

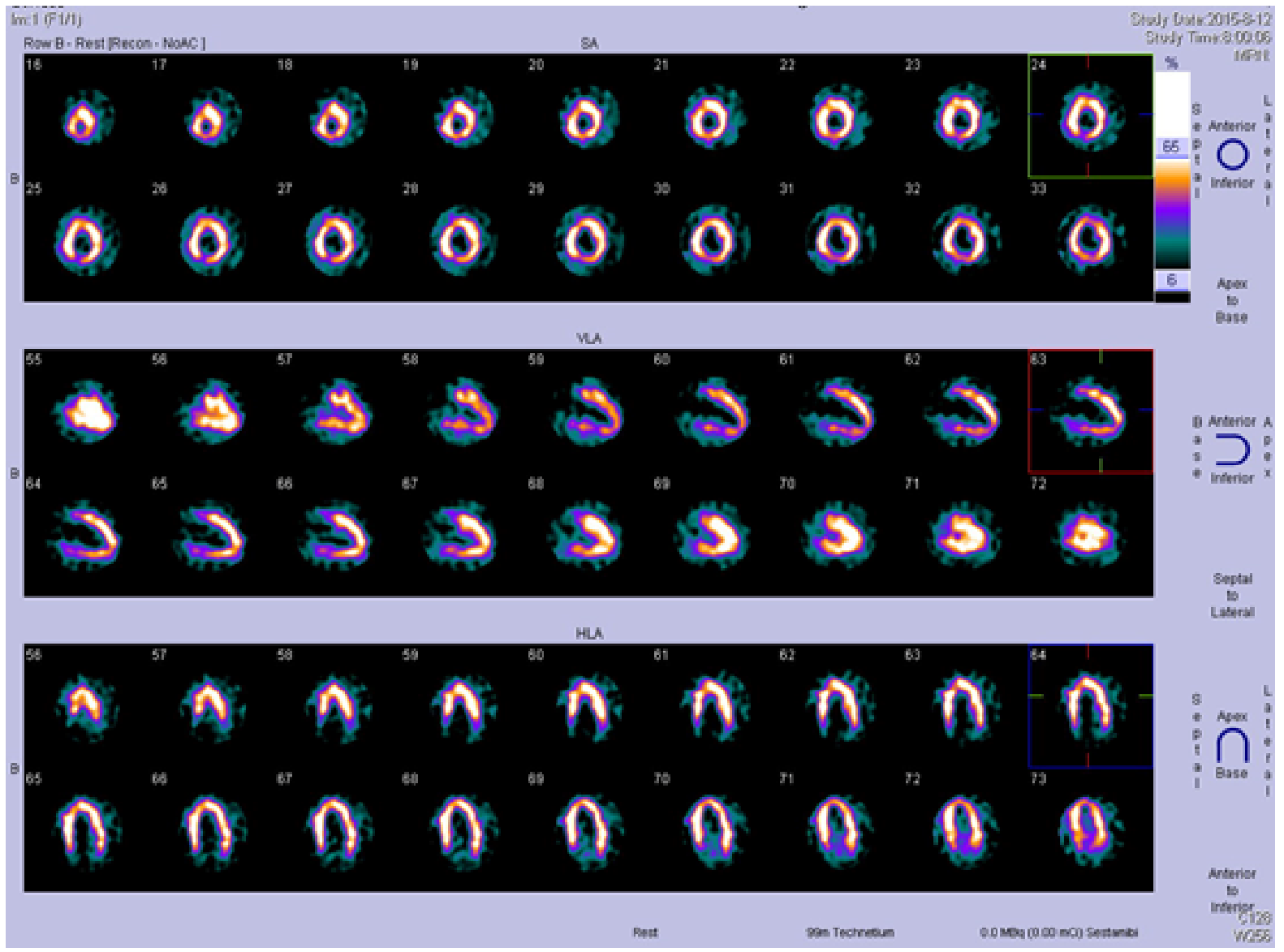

Figure 2. Single-photon emission computed tomography (SPECT), myocardial perfusion at 1 hour after intravenous injection of $99 \mathrm{mTc}-\mathrm{MIBI}$, revealed myocardial ischemia in the left ventricular inferior wall and the base of anterior wall. 

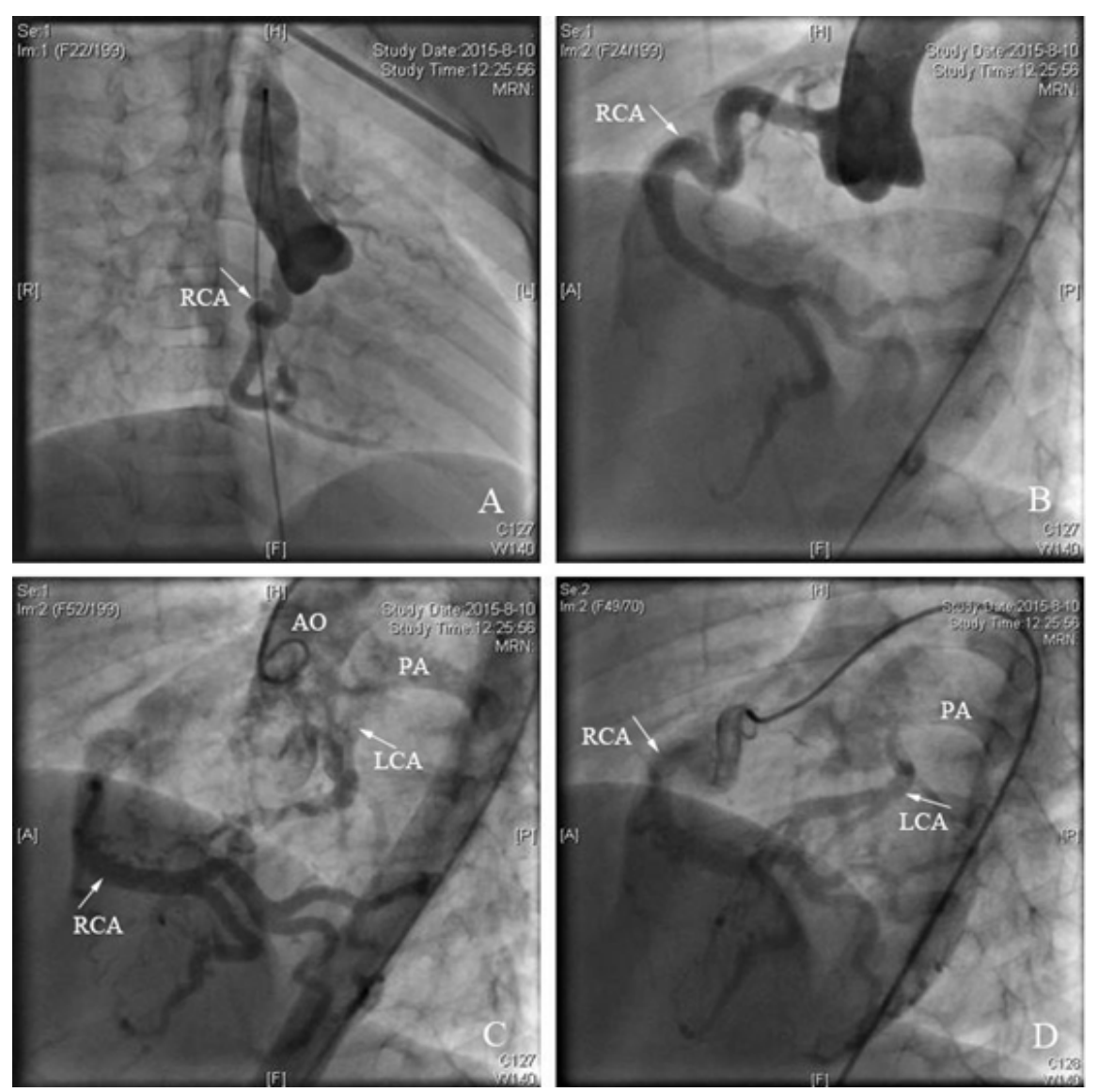

Figure 3. A: Antero-posterior position ascending aortography showed the dilated right coronary artery originated from the right sinus, but no coronary artery from the left sinus. B: Left anterior oblique ascending aortography showed the tortuous dilated right coronary artery originated from the right sinus, but no coronary artery from the left sinus. C: Left anterior oblique ascending aortography showed the right coronary artery connected to the left coronary artery by abundant collateral vessels, and the left coronary artery anomalously originated from the pulmonary artery. D: Selective right coronary angiography showed the contrast agent from the right coronary artery through the collateral vessels into the left coronary artery, and finally into the pulmonary artery.

RCA: right coronary artery; LCA: left coronary artery; AO: aorta; PA: pulmonary artery.

be confused when the flow forwards in the LCA in the case of ALCAPA with abundant collateral vessels fed by RCA. Although the diagnosis of ALCAPA is also possible with other non-invasive modalities such as cardiac CT or MR [9,10], the coronary angiography remains the gold standard method [3]. For ALCAPA, once the diagnosis is established, the surgical treatment should be taken into consideration in order to prevent further ischemia and necrosis of cardiac muscles. The direct reimplantation of the LCA onto the aorta is the current ideal option to achieve a definitive two-coronary artery anatomy and physiology and has a good short-term and long-term result $[11,12]$.

\section{References}

1. Wesselhoft H, Fawcet JS, Johnson AL (1968) Anomalous origin of the left coronary from the pulmonary trunk: its clinical spectrum, pathology and pathophysiology, based on a review of 140 cases with seven further cases. Circulation 38: 403-425. [Crossref]

2. Levitas A, Krymko H, Ioffe V, Zalzstein E, Broides A (2015) Anomalous left coronary artery from the pulmonary artery in infants and toddlers misdiagnosed as myocarditis. Pediatr Emerg Care 32: 232-234. [Crossref]

3. Rodriguez-Gonzalez M, Tirado AM, Hosseinpour R, de Soto JS (2015) Anomalous origin of the left coronary artery from the pulmonary artery: diagnoses and surgical results in 12 pediatric patients. Tex Heart Inst $J$ 42: 350-356. [Crossref]

4. Silverman NH (2015) Echocardiographic presentation of anomalous origin of the left coronary artery from the pulmonary artery. Cardiol Young 25: 1512-1523. [Crossref]

5. Yau JM, Singh R, Halpern EJ, Fischman D (2011) Anomalous origin of the left coronary artery from the pulmonary artery in adults: a comprehensive review of 151 adult cases and a new diagnosis in a 53-year-old woman. Clin Cardiol 34: 204-210. [Crossref]
6. Tseng KF (2013) Anomalous origin of left coronary artery from the pulmonary trunk in a mildly symptomatic adult female. Case Rep Surg 2013: 840741. [Crossref]

7. Dilaveris P, Koutagiar I, Alexopoulos N, Tsiachris D, Gatzoulis K (2014) Secondary prevention of sudden cardiac death in a 65 year untreated ALCAPA patient. Int $J$ Cardiol 176: e73-e74. [Crossref]

8. Cohen MS, Herlong RJ, Silverman NH (2010) Echocardiographic imaging of anomalous origin of the coronary arteries. Cardiol Young 20: 26-34. [Crossref]

9. Bruder O, Sabin GV, Barkhausen J (2005) Magnetic resonance imaging of anomalous origin of the left coronary artery from the pulmonary artery (Bland-White-Garland syndrome). Heart 91: 656. [Crossref]

10. Duan X, Yu T, Wang F, Liu H, Sun J, et al. (2015) Anomalous origin of the left coronary artery from the pulmonary artery in infants: imaging findings and clinical implications of cardiac computed tomography. J Comput Assist Tomogr 39: 189-195. [Crossref]

11. Ma K, Wang L, Hua Z, Yang K, Hu S, et al. (2015) Outcomes of coronary transfer for anomalous origin of the left coronary artery from the pulmonary artery. Eur $J$ Cardiothorac Surg 47: 659-664. [Crossref]

12. Cabrera AG, Chen DW, Pignatelli RH, Khan MS, Jeewa A, et al. (2015) Outcomes of anomalous left coronary artery from pulmonary artery repair: beyond normal function. Ann Thorac Surg 99: 1342-1347. [Crossref]

Copyright: (C2019 Ma X. This is an open-access article distributed under the terms of the Creative Commons Attribution License, which permits unrestricted use, distribution, and reproduction in any medium, provided the original author and source are credited. 